Old Dominion University

ODU Digital Commons

Electrical \& Computer Engineering Faculty

Publications

1982

\title{
Electrical and Structural Properties of $p$ - $n$ Junctions in cw Laser Annealed Silicon
}

\author{
M. Maier \\ D. Bimberg \\ G. Fernholz \\ H. Baumgart \\ Old Dominion University, hbaumgar@odu.edu \\ F. Phillipp
}

Follow this and additional works at: https://digitalcommons.odu.edu/ece_fac_pubs

Part of the Electronic Devices and Semiconductor Manufacturing Commons

\section{Original Publication Citation}

Maier, M., Bimberg, D., Fernholz, G., Baumgart, H., \& Phillipp, F. (1982). Electrical and structural properties of $p-n$ junctions in cw laser annealed silicon. Journal of Applied Physics, 53(8), 5904-5907. doi:10.1063/ 1.331432

This Article is brought to you for free and open access by the Electrical \& Computer Engineering at ODU Digital Commons. It has been accepted for inclusion in Electrical \& Computer Engineering Faculty Publications by an authorized administrator of ODU Digital Commons. For more information, please contact digitalcommons@odu.edu. 


\section{Electrical and structural properties of $p-n$ junctions in cw laser annealed silicon}

M. Maier, D. Bimberg, a) and G. Fernholz

Institut für Halbleitertechnik and Basislabor, SFB 202, RWTH Aachen, D-5100 Aachen, Federal Republic of

Germany

H. Baumgart ${ }^{\text {b) }}$

Max-Planck-Institut für Festkörperforschung, D-7000 Stuttgart, Federal Republic of Germany

F. Phillipp

Institut für Physik, Max-Planck-Institut für Metallforschung, D-7000 Stuttgart, Federal Republic of

Germany

(Received 12 January 1982; accepted for publication 20 March 1982)

Depth profiles of the electrical quality of ion implanted and $c w$ laser annealed $p-n$ junctions in silicon are obtained for the first time by secondary ion mass spectroscopy. A comparison with the crystallographic properties of the surface and the junction as observed by Nomarski optical microscopy as well as cross-sectional and plan view transmission electron microscopy is made. Samples containing slip dislocations show better insulation and a lower reverse bias current across the $p$ - $n$ junction as compared to samples with a perfect surface in agreement with current-voltage characteristics. Small dislocation loops located at the junction are found to degrade the junction quality.

PACS numbers: $81.40 . \mathrm{Rs}, 61.70 . \mathrm{Jc}, 79.20 . \mathrm{Ds}, 61.70 . \mathrm{Tm}$

Single crystalline silicon amorphized by ion implantation can be recrystallized by $\mathrm{cw}$ laser induced solid phase epitaxy (SPE).$^{1-3}$ In contrast to conventional furnace annealing and pulsed laser annealing, the depth distribution of the implanted dopant remains unaltered during $\mathrm{cw}$ laser annealing, ${ }^{1,2}$ a distinct advantage for many device applications.

Depending on laser power and dwell time, either optically perfect surface morphologies or surfaces showing slip lines are obtained. ${ }^{4.5}$ Slip lines are observed at high laser power or long dwell time and indicate plastic flow by the generation of slip dislocations due to the thermally induced stresses ${ }^{4,6}$ At first sight, samples with optically perfect surfaces might be preferred for device fabrication. This paper, however, presents evidence that inspection of surfaces is completely misleading and does not allow any predictions of the electrical quality of $p-n$ junctions produced by ion implantation and subsequent cw laser annealing. Secondary ion mass spectroscopy (SIMS) with an $\mathrm{O}_{2}^{+}$sputtering beam is applied for the first time to measure depth profiles of high resolution of the resistance of the successively removed depletion layer of $p-n$ junctions. The conclusions drawn from SIMS are compared to and agree with those drawn from current-voltage $(I-V)$ measurements. Samples with optically perfect surfaces show the lowest reverse bias resistance. These results are understood on the basis of cross-section transmission electron micrographs which show that the concentration of small disloaction loops close to the $p$ - $n$ junction is very high in samples without slip dislocations, degrading their electrical quality. In contrast, the influence of slip dislocations can be neglected.

(100)-oriented B-doped silicon specimens $\left(N_{A} \sim 2\right.$ $\times 10^{15} \mathrm{~cm}^{-3}$ ) are amorphized by implantation of $60 \mathrm{keV}$ ${ }^{75} \mathrm{As}$ ions at a dose of $7 \times 10^{15} \mathrm{~cm}^{-2}$. The specimens are

\footnotetext{
a) Permanent address: Institut für Festkörperphysik, TU Berlin, D-1000 Berlin, Federal Republic of Germany.

${ }^{b)}$ Present address: Bell Laboratories, Murray Hill, New Jersey 07974.
}

subsequently annealed by a cw argon ion laser without backside heating. The output power of the laser is $8 \mathrm{~W}$ and the spot diameter of the focussed laser beam is $40 \mu \mathrm{m}$. Various irradiation times are achieved by scanning the specimen with respect to the laser beam (scan width $30 \mu \mathrm{m}$ ) at controlled velocities. SIMS analyses are performed with an ATOMIKA instrument using both $\mathrm{O}_{2}^{+}$and $\mathrm{Ar}^{+}$primary ions of $10-\mathrm{keV}$ energy. The focussed and scanned $1 \mu \mathrm{A}$ primary ion beam forms a quadratic spot of $0.75 \mathrm{~mm} \times 0.75 \mathrm{~mm}$. The quadrupole mass filter of the SIMS instrument is tuned to the $\mathrm{Si}_{2}{ }^{+}$matrix molecular secondary ions. The dependence of the $\mathrm{Si}_{2}^{+}$secondary ion intensity on sputtering time is determined for the differently annealed samples. Transmission electron microscopy (TEM) studies are performed in an AEI-EM7 high voltage electron microscope operating at $1000 \mathrm{keV}$. The investigation of plan view as well as crosssection specimens permits determination of the nature and depth distribution of the crystal defects. Conventional guardring diodes are made of the annealed samples (see inset of Fig. 3) to determine the $I-V$ characteristics. The area of the $p$ - $n$ junction is $0.1 \mathrm{~mm}^{2}$. Ohmic contacts are made to the $n$ type region and to the $p$-type region using $\mathrm{Au}-\mathrm{Sb}$ alloy and $\mathrm{Al}$, respectively.

Figures $1(a)$ and 1 (b) show Nomarski optical micrographs of surfaces annealed with two different exposure times. The slip pattern in Fig. 1(a) (type A sample) is known to be generated by dislocation glide across the central region of the momentary laser spot. ${ }^{6}$ Using a laser dwell time of 50 msec suppresses generation of slip dislocations and yields samples with a nearly perfect surface morphology [Fig. 1(b), type B sample], seeming to be superior at first sight for applications.

The intensity of $\mathrm{Si}_{2}{ }^{+}$secondary ions is found to vary strongly with increasing depth (across the $p-n$ junction)-see Fig. 2-if sputtering is performed with $\mathrm{O}_{2}^{+}$primary ions. The depth profile of the implanted arsenic, also shown in Fig. 2, gives a rough idea of the width of the $n$-type part of the 


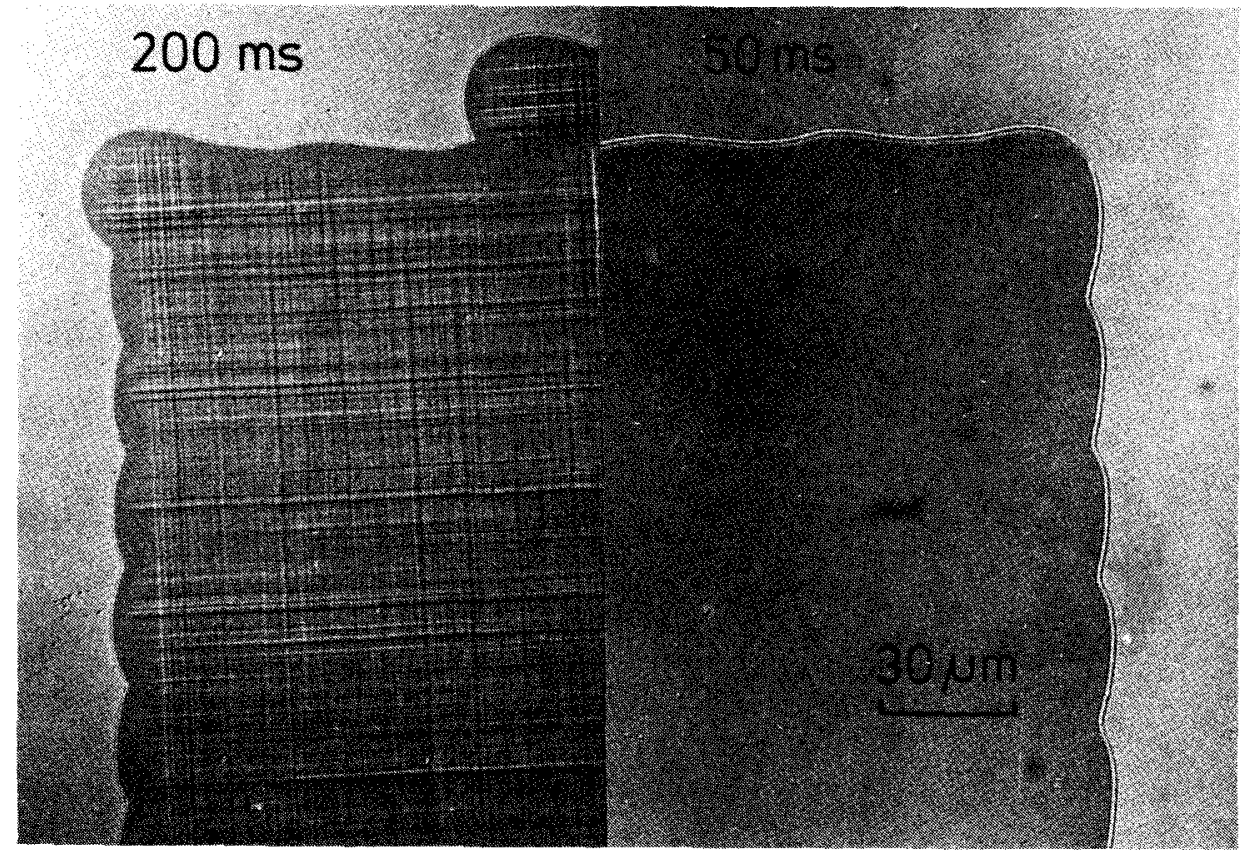

FIG. 1. Nomarski optical micrograph of solid phase annealed silicon showing (a) slip lines (200-msec exposure time) and (b) perfect surface morphology (50-msec exposure time).

otherwise $p$-type sample. The precise location of the $p$ - $n$ junction was determined by equating the implanted arsenic concentration with the boron contents of the substrate material.

The variation of the $\mathrm{Si}_{2}{ }^{+}$signal with depth is caused by a variation of the kinetic energy distribution function which shifts because of a depth dependent surface charging ${ }^{7}$ and does not result from a variation of the $\mathrm{Si}_{2}{ }^{+}$secondary ion yield. The detector of the SIMS instrument is equipped with an energy filter which allows only ions with a predetermined kinetic energy of small bandwidth to enter the quadrupole mass analyzer. If the sample bias is varied, ions with a correspondingly charged initial energy pass the energy filter. The

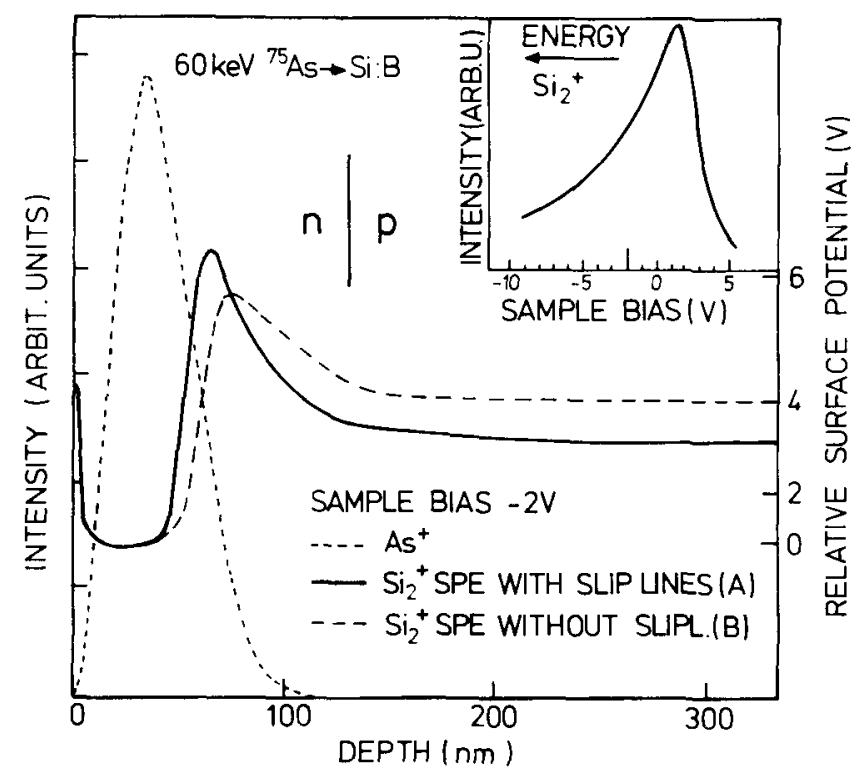

FIG. 2. Secondary ion signal intensity and relative surface potential vs depth of $60-\mathrm{keV}$ arsenic implanted and $\mathrm{cw}$ laser annealed silicon analyzed by oxygen primary ions. The inset shows the energy spectrum of $\mathrm{Si}_{2}^{+}$ions emitted from the substrate of a sample annealed without slip dislocations. inset of Fig. 2 shows an energy distribution of $\mathrm{Si}_{2}^{+}$ions sputtered from the $p$-type part of a type B sample. The energy distribution of the $n$-type layer is shifted by $4 \mathrm{~V}$. The surface of the $p$-type substrate during sputtering is apparently more positively charged by $4 \mathrm{~V}$ than the surface of the $n$-type layer. The surface potential difference is only $3.2 \mathrm{~V}$ for type $\mathrm{A}$ samples (see Fig. 2) due to a reduced charging of the $p$-type region. The SIMS intensity versus the sample bias dependence shown in the inset of Fig. 2 is used to calibrate the SIMS intensity variation in terms of a surface potential variation (right-hand vertical scale of Fig. 2). Remarkably enough, the surface potential shows a clear peak, up to $3 \mathrm{~V}$, for both types of samples on the $n$-side of the $p$ - $n$ junction and then falls off to values typical for the $p$-type substrate.

Let us briefly discuss the origin of the surface charge and its variation. Sputtering of silicon with $\mathrm{O}_{2}^{+}$priamry ions at normal incidence produces an almost perfect $\mathrm{SiO}_{2}$ layer. ${ }^{8}$ The existence of the insulating $\mathrm{SiO}_{2}$ layer is essential for the buildup of a stationary positive charge and surface potential. No surface potential variation is observed using $\mathrm{Ar}^{+}$as primary (sputtering) ion. Under quasiequilibrium sputtering conditions a constant current of $\sim 2 \mu \mathrm{A}$ flows through the insulating $\mathrm{SiO}_{2}$ layer and the rest of the sample assuring that no further charge buildup takes place. The resistance of the current path to ground of the part of the sample below the $\mathrm{SiO}_{2}$ layer governs the magnitude of the surface potential (and charge). From the physics of MOS devices it is known that a small positive bias of the metal contact is sufficient to cause accumulation of electrons at the interface between the $\mathrm{SiO}_{2}$ layer and $n$-type silicon. The sputtering beam itself induces a channel of higher conductivity through the $\mathrm{SiO}_{2}$ layer and the balancing current flow is maintained at modest surface potential. If the silicon underneath the $\mathrm{SiO}_{2}$ is $p$-type, an inversion layer followed by a zone completely depleted of charge carriers is formed upon positive charging of the $\mathrm{SiO}_{2}$. A much larger positive surface potential is needed to make the same current flow through this high resistivity zone in 
perfect agreement with our observations of an increase of the potential by 3.2 and $4 \mathrm{~V}$ for type $\mathrm{A}$ and $\mathrm{B}$ samples, respectively. The $p-n$ junction is reverse biased since the surface charge is positive. The surface potential first increases when the sputtering beam reaches the beginning of the depletion layer of the reverse biased $n-p$ junction and then peaks. Further sputtering leads to a decrease of the thickness of the depletion layer and results in a decreasing surface potential. At the actual position of the junction the surface potential has completely decreased to the bulk $p$-Si value as proved by Fig. 2. It should be reemphasized that the location of the junction is derived independently.

The difference $\Delta \varphi$ between the peak surface potential and its bulk value in the $p$-type region is a clear measure of the insulating properties of the $p-n$ junction under reverse bias conditions. $\Delta \varphi$ is a factor of two larger for type A samples than for type B samples. A much higher reverse bias current will flow in these samples than in type A samples at the same reverse bias. Apparently the slip dislocations depicted in Fig. 1 do not dominate the junction quality and an inspection of the surface morphology therefore does not give any conclusive information on the quality of the $p-n$ junction. On the other hand, the surface potential of type B samples is larger than that of type A samples for the regions below the $p-n$ junction, indicating a less resistive depletion layer for the latter samples (having slip dislocations).

Based on this very detailed knowledge of the depth profiles of the electrical quality of type A and B $p-n$ junctions,

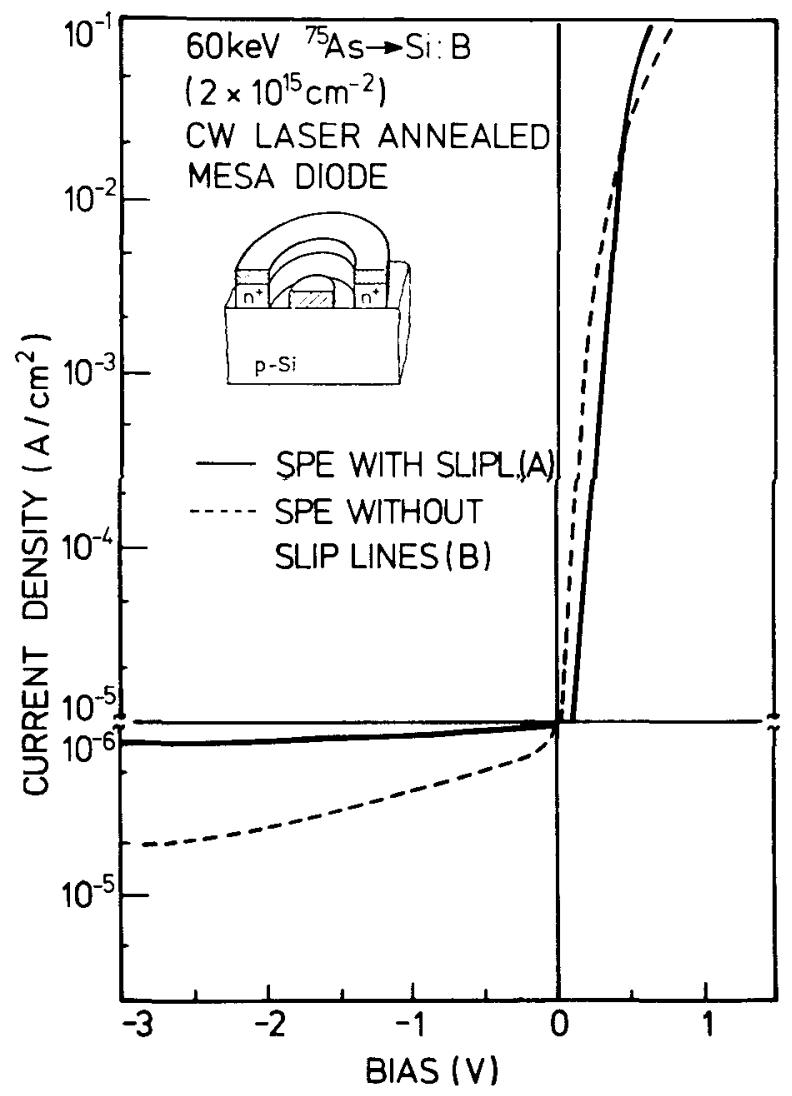

FIG. 3. $I$ - $V$ characteristics of a $p-n$ junction with slip dislocations (full curve) and without slip dislocations (broken curve). The inset shows the geometrical arrangement of the contacts. large differences between their $I-V$ characteristics are predicted and indeed observed. In Fig. 3 the $I-V$ characteristics of typical type $A$ and type $B$ samples are compared with each other. The reverse bias current is more than a factor of three larger and the forward current at small voltages is approximately an order of magnitude larger in the type B samples than in the type A samples. Thus the $I-V$ characteristics of $p$ $n$ junctions crossed by slip dislocations are closer to ideal than the characteristics of junctions without slip dislocations.

Electron microscopy studies indeed reveal that the optically observed surface slip lines are not indicative of the defect structure in the $p-n$ junction region. The cross-sectional electron micrograph of a type A sample in Fig. 4(a) shows that the dislocations penetrating the surface and generating the slip lines are part of a dislocation network located deeply below the specimen surface. The crystalline quality of the $p$ -
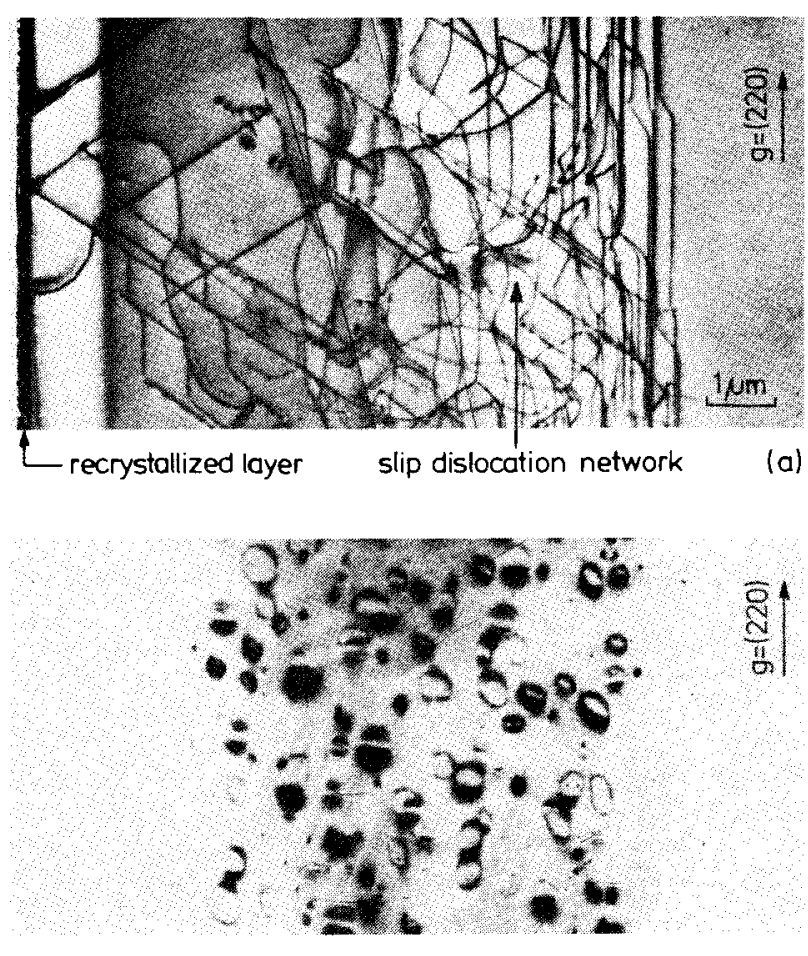

(b)

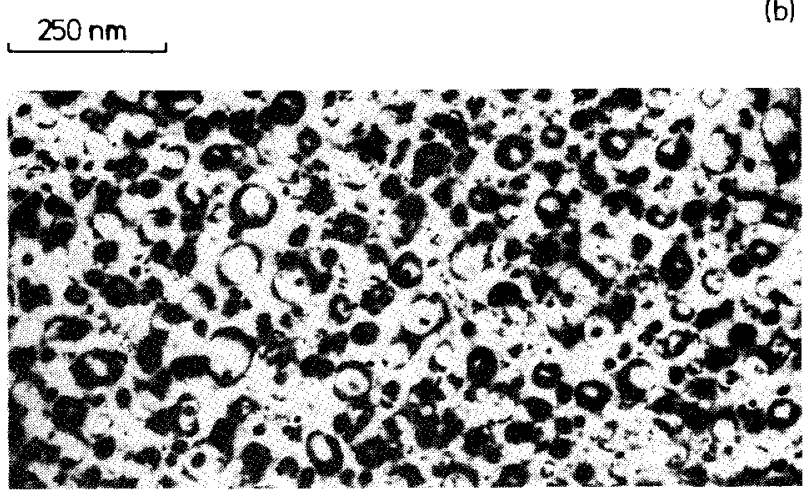

(c)

FIG. 4. (a) Cross-section TEM micrograph showing slip dislocation network and recrystallized layer containing small dislocation loops. (b) Weak beam plan view micrograph of a sample containing slip dislocations (type A) and (c) containing no slip dislocations (type B). 
$n$ junction region, however, is essentially determined by small interstitial type dislocation loops that are found in both types of samples. ${ }^{9}$ The dislocation density corresponding to these loops is more than two orders of magnitude higher than the density of slip dislocations penetrating the $p$ $n$ junction $\left(-4 \times 10^{7} \mathrm{~cm}^{-2}\right)$. Thus, obviously the influence of the slip dislocations on the electrical behavior of the $p-n$ junction is negligible compared to the influence of the dislocation loops. As determined by the investigation of plan view specimens containing the regrown layer, the mean loop density in type A samples is less by a factor of 3 than in type B samples [Fig. 4(b), 4(c)]. Only loops are visible in the area depicted in Figs. $4(\mathrm{~b})$ and $4(\mathrm{c})$. The loops are arranged in stripes being parallel to the scan direction. An approximately equal area between the stripes is completely free of loops in type A samples [Fig. 4(b)]. Apparently the decrease of the loop density due to the longer annealing is responsible for the better $p-n$ junction quality of the type $\mathrm{A}$ samples. The higher surface potential sustained at depths $>200 \mathrm{~nm}$ of type $B$ samples on the other hand is due to the absence of slip dislocations.

In conclusion, we have shown for the first time that depth profiles of the electrical quality of ion implanted and $\mathrm{cw}$ laser annealed $p-n$ junctions in silicon can be obtained by SIMS with $\mathrm{O}_{2}^{+}$primary ions and yield results which are opposite to conclusions one might draw from optical micrographs. The SIMS investigations, additional $I$ - $V$ characteristics, and TEM micrographs give clear evidence that the electrical quality of As-implanted cw laser annealed $p-n$ junctions is dominated by small dislocation loops located at the junction and not by slip dislocations penetrating the junctions.

\section{ACKNOWLEDGMENTS}

We are indebted to D. R. Young for helpful discussions, to $R$. Uebbing for his support in annealing the samples, and to U. Weimer for preparing the mesa diodes. Part of this work has been supported under the technological program of the Federal Department of Research and Technology (BMFT) of the FRG.

\footnotetext{
${ }^{1}$ A. Gat, J. F. Gibbons, T. J. Magee, J. Peng, V. R. Deline, P. Williams, and C. A. Evans, Jr., Appl. Phys. Lett. 32, 276 (1978).

${ }^{2}$ J. S. Williams, W. L. Brown, H. J. Leamy, J. M. Poate, J. W. Rogers, D. Rousseau, G. A. Rozgonyi, J. A. Schelnutt, and T. T. Scheng, Appl. Phys. Lett. 33, 542 (1978).

${ }^{3}$ D. H. Auston, J. A. Golovchenko, P. R. Smith, C. M. Surko, and T. N. C. Venkatesan, Appl. Phys. Lett. 33, 539 (1978).

${ }^{4}$ G. A. Rozgonyi and H. Baumgart, J. Phys. (Paris) 41, Suppl. 5, C 4-85 (1980).

${ }^{5}$ K. Ishida, H. Okabayashi, and M. Yoshida, Appl. Phys. Lett. 37, 175 (1980).

${ }^{6}$ H. Baumgart, F. Phillipp, G. A. Rozgonyi, and U. Gösele, Appl. Phys. Lett. 38, 95 (1981).

${ }^{7}$ M. Maier, D. Bimberg, H. Baumgart, and F. Phillipp, "Secondary Ion Mass Spectrometry SIMS III," in Springer Series in Chemical Physics, edited by A. Bennighoven, J. Giber, J. László, M. Riedel, and H. W. Werner (Springer, Berlin, 1982), Vol. 19, p. 336.

${ }^{8}$ W. Reuter and K. Wittmaack, Appl. Surf. Sci. 5, 221 (1980).

${ }^{9}$ H. Baumgart, O. Hildebrand, F. Phillipp, and G. A. Rozgonyi, Inst. Phys. Conf. Ser. No. 60, 127 (1981).
} 